\title{
HARNACK INEQUALITY FOR HYPOELLIPTIC SECOND ORDER PARTIAL DIFFERENTIAL OPERATORS
}

\author{
ALESSIA E. KOGOJ AND SERGIO POLIDORO
}

Abstract. We consider nonnegative solutions $u: \Omega \longrightarrow \mathbb{R}$ of second order
hypoelliptic equations
\[ \mathscr{L} u(x)=\sum_{i, j=1}^{n} \partial_{x_{i}}\left(a_{i j}(x) \partial_{x_{j}} u(x)\right)+\sum_{i=1}^{n} b_{i}(x) \partial_{x_{i}} u(x)=0, \] where $\Omega$ is a bounded open subset of $\mathbb{R}^{n}$ and $x$ denotes the point of $\Omega$. For any fixed $x_{0} \in \Omega$, we prove a Harnack inequality of this type

$$
\sup _{K} u \leq C_{K} u\left(x_{0}\right) \quad \forall u \text { s.t. } \mathscr{L} u=0, u \geq 0,
$$

where $K$ is any compact subset of the interior of the $\mathscr{L}$-propagation set of $x_{0}$ and the constant $C_{K}$ does not depend on $u$.

\section{INTRODUCTION}

We consider second order partial differential operators $\mathscr{L}$ acting on functions $u \in C^{2}(\Omega)$ as follows

$$
\mathscr{L} u(x):=\sum_{i, j=1}^{n} \partial_{x_{i}}\left(a_{i j}(x) \partial_{x_{j}} u(x)\right)+\sum_{i=1}^{n} b_{i}(x) \partial_{x_{i}} u(x)
$$

for $x$ belonging to any open bounded subset $\Omega$ of $\mathbb{R}^{n}$. The coefficients $a_{i j}, b_{i}$ are real functions and belong to $C^{\infty}(\bar{\Omega})$ for $1 \leq i, j \leq n$. Moreover, $A:=\left(a_{i j}\right)$ is a $n \times n$ symmetric and non-negative matrix. We also assume the following hypotheses:

(H1) $\mathscr{L}-\beta$ and $\mathscr{L}^{*}$ are hypoelliptic for every constant $\beta \geq 0$;

(H2) $\inf _{\Omega} a_{11}>0$.

We recall that $\mathscr{L}$ is said hypoelliptic if every distribution $u$ in $\Omega$ such that $\mathscr{L} u \in$ $C^{\infty}(\Omega)$ is a smooth function. We note that condition (H2) ensures that for every $x \in \Omega$ there exists $\xi \in \mathbb{R}^{n}$ such that $\langle A(x) \xi, \xi\rangle>0$ that is $\mathscr{L}$ is non-totally degenerate, in accordance with Definition 5.1 in Bon69. We can drop condition (H2) if the operator $\widetilde{\mathscr{L}}=\partial_{x_{n+1}}^{2}+\mathscr{L}$ acting on $\mathbb{R}^{n+1}$ satisfies (H1) (see Corollary 2).

2010 Mathematics Subject Classification. 35H10; 35K10; 31D05.

Key words and phrases. Harnack inequality, Hypoelliptic operators, Potential theory. 
The main result of this paper is the following Harnack inequality for the nonnegative solutions of the equation $\mathscr{L} u=0$. It obviously applies to the Laplacian and to the heat operators and in these cases it restores the classical elliptic and parabolic Harnack inequalities.

Theorem 1. Assume that $\mathscr{L}$ statisfies (H1) and (H2). Let $x_{0}$ in $\Omega$ and let $K$ be any compact set contained in the interior of $\overline{\mathscr{P}\left(x_{0}, \Omega\right)}$, then there exists a positive constant $C=C\left(x_{0}, K, \Omega, \mathscr{L}\right)$ such that

$$
\sup _{K} u \leq C u\left(x_{0}\right)
$$

for every non-negative solution $u$ of $\mathscr{L} u=0$ in $\Omega$.

We introduce here the definition of $\mathscr{L}$-propagation set $\mathscr{P}\left(x_{0}, \Omega\right)$ appearing it the above statement. It is the set of all points $x$ reachable from $x_{0}$ by a propagation path:

$$
\mathscr{P}\left(x_{0}, \Omega\right):=\left\{x \in \Omega \mid \exists \gamma \mathscr{L} \text {-propagation path, } \gamma(0)=x_{0}, \gamma(T)=x\right\}
$$

A $\mathscr{L}$-propagation path is any absolutely continuous path $\gamma:[0, T] \longrightarrow \Omega$ such that

$$
\gamma^{\prime}(t)=\sum_{j=1}^{n} \lambda_{j}(t) X_{j}(\gamma(t))+\mu(t) Y(\gamma(t)) \quad \text { a.e. in }[0, T]
$$

for suitable piecewise constant real functions $\lambda_{1}, \ldots, \lambda_{n}$, and $\mu, \mu \geq 0$. $X_{1}(x), \ldots, X_{n}(x), Y(x)$ are the vector fields defined in the following way:

$$
X_{j}(x):=\sum_{i=1}^{n} a_{j i}(x) \partial_{x_{i}}, \quad j=1, \ldots, n, \quad Y(x):=\sum_{i=1}^{n} b_{i}(x) \partial_{x_{i}} .
$$

As we said at the beginning of the Introduction, our main result can holds also under somehow weaker assumptions on $\mathscr{L}$. Only in the following Corollary the hypotheses (H1) and (H2) on $\mathscr{L}$ are replaced by the assumption that the operator $\widetilde{\mathscr{L}}=\partial_{x_{n+1}}^{2}+\mathscr{L}$ in $\mathbb{R}^{n+1}$ satisfies $(\mathrm{H} 1)$. Of course if $\widetilde{\mathscr{L}}$ satisfies assumption (H1) then also $\mathscr{L}$ does. A simple example of operator satisfying the hypotheses of this Corollary but not the ones of Theorem 1 is $\partial_{x_{1}}=x_{1}^{2} \partial_{x_{2}}^{2}$ in $\mathbb{R}^{2}$.

Corollary 2. If the operator $\widetilde{\mathscr{L}}=\partial_{x_{n+1}}^{2}+\mathscr{L}$ acting on $\Omega \times \mathbb{R}$ satisfies (H1) then for every $x_{0}$ in $\Omega$ and for every compact set $K$ contained in the interior of the $\mathscr{L}$-propagation set $\overline{\mathscr{P}\left(x_{0}, \Omega\right)}$, there exists a positive constant $C=C\left(x_{0}, K, \Omega, \mathscr{L}\right)$ such that

$$
\sup _{K} u \leq C u\left(x_{0}\right)
$$

for every non-negative solution $u$ of $\mathscr{L} u=0$ in $\Omega$. 
The notion of propagation set $\mathscr{P}\left(x_{0}, \Omega\right)$ has been introduced by Amano in his work on maximum principle (see [Ama79, Theorem 2]). In our case it reads as follows.

Assume that $u$ is a (smooth) solution of $\mathscr{L} u=0$ in $\Omega$. If $u$ attains its maximum at a point $x_{0}$ in $\Omega$, then $u \equiv u\left(x_{0}\right)$ in $\overline{\mathscr{P}\left(x_{0}, \Omega\right)}$.

The Amano maximum principle is a crucial tool to prove Theorem 1 using the abstract Harnack inequality of the Potential Theory ([CC72, Proposition 6.1.5]). We observe that Harnack inequalities based on results of Potential Theory were proved in [BLU02, Theorem 4.2] for heat equations on Carnot groups and in CNP10, Theorem 1.1] and in [CMP15, Theorem 5.2] for more general evolution equations. The class of hypoelliptic operators considered in [CNP10, CMP15] is

$$
\sum_{j=1}^{m} \widetilde{X}_{j}^{2}(y)+\widetilde{X}_{0}(y)-\partial_{t}
$$

where $\widetilde{X}_{j}$ are smooth vector fields on $\mathbb{R}^{N}$ and $(y, t)$ denoted the point of any subset of $\mathbb{R}^{N+1}$. We explicitly note that operator (1.3) is a particular example of the operators (1.1), with respect to the variable $x=(y, t)$. In both papers the operators are assumed left translation invariant w.r.t. a Lie group in $\mathbb{R}^{N+1}$ and endowed with a global fundamental solution. We point out that the use of the fundamental solution is a key step in verifying the separation axiom of the axiomatic Potential Theory.

The approach used in this note allows us to prove the validity of the separation axiom in Section 2.4 without requiring the existence of any global fundamental solution on every bounded open set. We rely only on hypoellipticity, on non total degeneracy of $\mathscr{L}$ and the following maximum principle due to Picone that we recall for the sake of completeness.

Let $V$ be any open (bounded) subset of $\Omega$. Assume that exists a function $w$ : $V \rightarrow \mathbb{R}$ such that $\mathscr{L} w<0$ in $V$ and $\inf _{V} w>0$. Then for every $u \in C^{2}(V)$ such that

$$
\mathscr{L} u \geq 0 \quad \text { in } V, \quad \limsup _{x \rightarrow \xi} u(x) \leq 0 \quad \forall \xi \in \partial V,
$$

we have $u \leq 0$ in $V$.

In our case the existence of a function $w$ follows from (H2) and from the smoothness of the coefficients. Indeed, under these assumptions, we can choose two positive real constants $M$ and $\lambda$ such that the function

$$
w(x)=w\left(x_{1}, \ldots, x_{N}\right)=M-e^{\lambda x_{1}}
$$

has the required properties. 
This paper is organized as follows. In Section 2 all the notions and results from Potential Theory that we need are briefly recalled. In Section 3 we show that the set of the solutions $u$ of $\mathscr{L} u=0$ in $\Omega$ satisfies the axioms of the Doob Potential Theory. In Section 4 we prove that the $\mathscr{L}$-propagation set of $x_{0}$ is a subset of the smallest absorbent set containing $x_{0}$. In this way we derive the Harnack inequality for the non-negative solutions $u$ of $\mathscr{L} u=0$. In Section 5 the propagation sets of some meaningful operators are studied. In particular we focus on the following operators: $\partial_{x_{1}}^{2}+x_{1} \partial_{x_{2}}$ in $\mathbb{R}^{2}$ and $\partial_{x_{1}}^{2}+\sin \left(x_{1}\right) \partial_{x_{2}}+\cos \left(x_{1}\right) \partial_{x_{3}}$ in $\mathbb{R}^{3}$, and we show that the geometry of the relevant Harnack inequality may appear either of parabolic or elliptic-type, depending on the choice of $\Omega$, even if both operators are parabolic.

\section{Some Rechlls from Potential Theory}

We recall some definitions and results of the Potential Theory that we need to prove our Harnack inequality. For a detailed description of the general theory of harmonic spaces we refer to [BLU07, chapter 6], [CC72] and to Bau66].

\subsection{Sheafs of functions and harmonic sheafs in $\Omega$.}

Let $V$ be any open subset of $\Omega$. We denote by $\overline{\mathbb{R}}$ the set $\mathbb{R} \cup\{\infty,-\infty\}$ and by $\overline{\mathbb{R}}^{V}$ the set of functions $u: V \longrightarrow \overline{\mathbb{R}}$. Moreover $C(V, \mathbb{R})$ is the vector space of real continuous functions defined on $V$.

A map

$$
\mathscr{F}: V \mapsto \mathscr{F}(V) \subseteq \overline{\mathbb{R}}^{V}
$$

is a sheaf of functions in $\Omega$ if

(i) $V_{1}, V_{2} \subseteq \Omega, V_{1} \subseteq V_{2},\left.u \in \mathscr{F}\left(V_{2}\right) \Longrightarrow u\right|_{V_{1}} \in \mathscr{F}\left(V_{1}\right)$;

(ii) $V_{\alpha} \subseteq \Omega \forall \alpha \in \mathcal{A}, u: \bigcup_{\alpha \in A} V_{\alpha} \longrightarrow \overline{\mathbb{R}},\left.u\right|_{V_{\alpha}} \in \mathscr{F}\left(V_{\alpha}\right) \Longrightarrow u \in \mathscr{F}\left(\bigcup_{\alpha \in \mathcal{A}} V_{\alpha}\right)$.

When $\mathscr{F}(V)$ is a linear subspace of $C(V, \mathbb{R})$ for every $V \subseteq \Omega$, we say that the sheaf of functions $\mathscr{F}$ on $V$ is harmonic and we denote it $\mathcal{H}(\Omega)$.

\subsection{Regular open sets, harmonic measures and absorbent sets.}

Let $\mathcal{H}$ be a harmonic sheaf on $\Omega$. We say that an open set $V \subseteq \Omega$ is regular if:

(i) $\bar{V} \subseteq \Omega$ is compact and $\partial V \neq \emptyset$;

(ii) for every continuous function $\varphi: \partial V \longrightarrow \mathbb{R}$, there exists a unique function in $\mathcal{H}(V)$, that we denote by $h_{\varphi}^{V}$, such that $h_{\varphi}^{V}(x) \underset{x \rightarrow \xi}{\longrightarrow} \varphi(\xi)$ for every $\xi \in \partial V$

(iii) if $\varphi \geq 0$ then $h_{\varphi}^{V} \geq 0$.

From $(i i)$ and $($ iii $)$ it follows that, for every regular set $V$ and for every $x \in V$, the map

$$
C(\partial V) \ni \varphi \longmapsto h_{\varphi}^{V}(x) \in \mathbb{R}
$$


is linear and positive. Thus, the Riesz representation theorem (see e.g. [Rud87]), implies that, for every regular set $V$ and for every $x \in V$, there exists a regular Borel measure, that we denote by $\mu_{x}^{V}$, supported in $\partial V$, such that

$$
h_{\varphi}^{V}(x)=\int_{\partial V} \varphi(y) d \mu_{x}^{V}(y) \quad \forall \varphi \in C(\partial V) .
$$

The measure $\mu_{x}^{V}$ is called the harmonic measure related to $V$ and $x$.

Now, let $A$ be a closed subset of $\Omega$. We say that $A$ is absorbent if it contains the supports of all the harmonic measures related to its points. More precisely,

for every $x \in A$ and every regular set $V$ containing $x, \operatorname{supp} \mu_{x}^{V} \subseteq A$.

If $x_{0} \in \Omega$, we define $\Omega_{x_{0}}$ as the smallest absorbent set containing $x_{0}$ :

$$
\Omega_{x_{0}}:=\bigcap_{\substack{A \text { absorbent } \\ A \ni x_{0}}} A .
$$

\subsection{Superharmonic functions.}

A function $u: \Omega \longrightarrow]-\infty, \infty]$ is called superharmonic in $\Omega$ if

(i) $u$ is lower semi-continuous;

(ii) for every regular set $V, \bar{V} \subseteq \Omega$, and for every $\varphi \in C(\partial V, \mathbb{R}), \varphi \leq\left. u\right|_{\partial \Omega}$, it follows $u \geq h_{\varphi}^{V}$ in $V$;

(iii) the set $\{x \in \Omega \mid u(x)<\infty\}$ is dense in $\Omega$.

We denote by $\mathscr{S}(\Omega)$ the family of the superharmonic functions on $\Omega$.

By the maximum principle, we have that every function $u \in C^{2}(\Omega)$ such that $\mathscr{L} u \leq 0$ in $\Omega$ is superharmonic (see [BLU07, Proposition 7.2.5]).

\subsection{Doob harmonic spaces and Harnack inequality.}

We say that a harmonic sheaf $\mathcal{H}(\Omega)$ is a Doob harmonic space if the following axioms are satisfied.

(A1) Positivity axiom:

For every $x \in \Omega$, there exists an open set $V \ni z$ and a function $u \in \mathcal{H}(V)$ such that $u(x)>0$.

(A2) Doob convergence axiom:

Let $\left(u_{n}\right)_{n \in \mathbb{N}}$ be a monotone increasing sequence in $\mathcal{H}(\Omega)$ and let $u:=\sup _{n \in \mathbb{N}} u_{n}$. If the set $\{x \in \Omega \mid u(x)<\infty\}$ is dense in $\Omega$, then $u \in \mathcal{H}(\Omega)$.

(A3) Regularity axiom:

There is a basis of the euclidean topology of $\Omega$ formed by regular sets.

(A4) Separation axiom:

$\mathscr{S}(\Omega)$ separates the points of $\Omega$ in this sense: for every $y$ and $z$ in $\Omega$, 
$y \neq z$, there exist two non-negative functions $u$ and $v$ in $\mathscr{S}(\Omega)$ such that $u(y) v(z) \neq u(z) v(y)$.

We close the section recalling that in this setting the abstract Harnack inequality from the Parabolic Potential Theory holds [CC72, Proposition 6.1.5].

Theorem A. Let $(\Omega, \mathcal{H})$ be a Doob harmonic space, $x_{0} \in \Omega$ and let $K$ be a compact set contained in the interior of $\Omega_{x_{0}}$, the smallest absorbent set containing $x_{0}$. Then there exists a positive constant $C=C\left(x_{0}, K, \Omega\right)$ such that

$$
\sup _{K} u \leq C u\left(x_{0}\right) \quad \forall u \in \mathcal{H}(\Omega), u \geq 0 .
$$

\section{The harmonic SPACE of the solutions of $\mathscr{L} u=0$}

We show that the set of the solutions of the equation $\mathscr{L} u=0$ is a Doob harmonic space in $\Omega$. For every $V \subseteq \Omega$ we consider the harmonic sheaf

$$
\mathbb{R}^{n} \supseteq V \longmapsto \mathcal{H}(V)
$$

where

$$
\mathcal{H}(V)=\left\{u \in C^{\infty}(V) \mid \mathscr{L} u=0\right\}
$$

and $\mathscr{L}$ is the operator (1.1).

The positivity axiom (A1) is plainly verified. Indeed every constant function belongs to $\mathcal{H}(\Omega)$.

(A2) is a consequence of a weak Harnack inequality due to Bony (see Bon69, Theoreme 7.1]); see also [KL04, Proposition 7.4]).

(A3), i.e. the existence of a basis of the euclidean topology of $\Omega$ formed by regular sets, can be proved as in Bon69, Corollarie 5.2], see also [BLU07, Proposition 7.1.5]. We stress that the tools used in its proof are only the hypoellipticity, the non totally degeneracy of the operator $\mathscr{L}$ and the classical Picone Maximum Principle.

Now we are left to verify the separation axiom (A4). As in our setting the constant are superharmonic functions, we need to prove that

$$
\forall y, z \in \Omega, y \neq z, \exists u \in \mathscr{S}(\Omega), u \geq 0 \text {, such that } u(y) \neq u(z) .
$$

Now, let $y=\left(y_{1}, \ldots, y_{n}\right)$ and $z=\left(z_{1}, \ldots, z_{n}\right)$ be two different points in $\Omega$.

We observe that the function $w(x)=w\left(x_{1}, \ldots, x_{N}\right)=M-e^{\lambda x_{1}}$, as in (1.4), for suitable real positive constants $\lambda$ and $M$, is non-negative and $\mathscr{L} w(x)<0$ for every $x \in \Omega$, hence $w \in \mathscr{S}(\Omega)$.

If $y_{1} \neq z_{1}$, we can choose $u(x)=w(x)$ to separate $y$ and $z$ and we are done. 
If $y_{1}=z_{1}$, we set $u(x)=|x-y|^{2}+w(x)$. Also in this case, for suitable $\lambda$ and $M, u$ is non-negative, $u \in C^{2}(\Omega)$ and $\mathscr{L} u(x)=\mathscr{L}\left(|x-y|^{2}\right)+\mathscr{L}(w(x))<0$ in $\Omega$. Moreover $u(y)-u(z)=|z-y|^{2}$, so (3.1) is satisfied.

\section{Propagation sets and Harnack inequality}

Let $X_{1}(x), \ldots, X_{n}(x), Y(x)$ be the vector fields defined in the following way:

$$
\begin{aligned}
& X_{i}(x)=\sum_{j=1}^{n} a_{i j}(x) \partial_{x_{j}}, \quad 1 \leq i \leq n, \\
& Y(x)=\sum_{i=1}^{n} b_{i}(x) \partial_{x_{i}} .
\end{aligned}
$$

We recall that a $\mathscr{L}$-propagation path is any absolutely continuous path $\gamma$ : $[0, T] \longrightarrow \Omega$ such that

$$
\gamma^{\prime}(t)=\sum_{j=1}^{n} \lambda_{j}(t) X_{j}(\gamma(t))+\mu(t) Y(\gamma(t)) \quad \text { a.e. in }[0, T]
$$

for suitable piecewise constant real functions $\lambda_{1}, \ldots, \lambda_{n}$, and $\mu$ with $\mu \geq 0$.

For a point $x_{0}$ in $\Omega$, we define the $\mathscr{L}$-propagation set as the set of all points $x$ such that $x$ and $x_{0}$ can be connected by a propagation path, running from $x_{0}$ to $x$ :

$\mathscr{P}\left(x_{0}, \Omega\right):=\left\{x \in \Omega \mid \exists \gamma:[0, T] \rightarrow \Omega, \gamma \mathscr{L}\right.$-propagation path, $\left.\gamma(0)=x_{0}, \gamma(T)=x\right\}$.

Proceeding as in [CMP15, Lemma 5.8], we prove now that the $\mathscr{L}$-propagation set of $x_{0}$ is a subset of every absorbent set containing $x_{0}$. This Lemma, based on the maximum propagation principle, is a key lemma in order to get our Harnack inequality so we prefer to give here its detailed proof.

Lemma 2. For every $x_{0}$ in $\Omega, \mathscr{P}\left(x_{0}, \Omega\right) \subseteq \Omega_{x_{0}}$.

Proof. By contradiction, suppose $x \in \mathscr{P}\left(x_{0}, \Omega\right)$ and $x \notin \Omega_{x_{0}}$. There exists an absolutely continuous path $\gamma$ connecting $x_{0}$ and $x$ :

$$
\gamma:[0, T] \longrightarrow \Omega, \quad \gamma(0)=x_{0}, \quad \gamma(T)=x .
$$

As $\Omega_{x_{0}}$ is a subset closed in $\Omega$ and $\gamma$ is continuous, there will be a time $t_{1}$ such that $\gamma\left(t_{1}\right)=x_{1} \in \Omega_{x_{0}}$ and $\gamma(t) \notin \Omega_{x_{0}}$ when $t$ is in $\left.] t_{1}, T\right]$.

Let's take a regular open set $V$ containing $x_{1}$. There will be $\left.\left.t_{2} \in\right] t_{1}, T\right]$ such that $x_{2}=\gamma\left(t_{2}\right) \in \partial V$. From what we wrote before, $x_{2}$ does not belong to $\Omega_{x_{0}}$.

Take now a neighborhood of $x_{2}, U$ such that $U \cap \partial V \subseteq \Omega \backslash \Omega_{x_{0}}$ and consider a function $\varphi$ defined on $\partial V$ such that $\varphi$ is strictly positive in $U \cap \partial V$ and 0 otherwise. 


$$
h_{\varphi}^{V}\left(x_{1}\right)=\int_{\partial V} \varphi(y) d \mu_{x_{1}}^{V}(y)=\int_{U \cap \partial V} \varphi(\zeta) d \mu_{x_{1}}^{V}(\zeta)=0,
$$

because $x_{1}$ is in $\Omega_{x_{0}}$ and supp $\mu_{x_{1}}^{V} \subseteq \Omega_{x_{0}}$ for every regular set $V$. But $h_{\varphi}^{V}$ is nonnegative and it would attain its minimum at $x_{1}$. From Amano minimum propagation principle [Ama79, Theorem 2], it would follow that

$$
\left.h_{\varphi}^{V}(\gamma(t))=0 \quad \forall t \in\right] t_{1}, t_{2}[.
$$

In conclusion, we would have that

$$
h_{\varphi}^{V}(x) \underset{x \rightarrow x_{2}}{\longrightarrow} \varphi\left(x_{2}\right)>0
$$

and

$$
h_{\varphi}^{V}(\gamma(t)) \underset{t \rightarrow t_{2}^{-}}{\longrightarrow} h_{\varphi}^{V}\left(\gamma\left(t_{2}\right)\right)=0
$$

that is a contraddiction.

We are now ready to give the proofs of our main results.

Proof of Theorem 1, Let $x_{0}$ in $\Omega$ and let $K$ be a compact set contained in the interior of $\overline{\mathscr{P}\left(x_{0}, \Omega\right)}$. As $\Omega_{x_{0}}$ is a closed subset of $\Omega$, Lemma 2 implies that $\overline{\mathscr{P}\left(x_{0}, \Omega\right)} \subseteq \Omega_{x_{0}}$. On the other hand, as we showed in Section 3 , the set of the solutions of the equation $\mathscr{L} u=0$ is a Doob harmonic space in $\Omega$. Then, by Theorem $\mathrm{A}$, there exists a positive constant $C=C\left(x_{0}, K, \Omega, \mathscr{L}\right)$ such that

$$
\sup _{K} u \leq C u\left(x_{0}\right)
$$

for every non-negative solution $u$ of $\mathscr{L} u=0$ in $\Omega$.

Proof of Corollary 圆. We set $\left.\widetilde{x}:=\left(x, x_{n+1}\right), \widetilde{\Omega}:=\Omega \times\right]-1,1\left[, \widetilde{K}:=K \times\left[-\frac{1}{2}, \frac{1}{2}\right]\right.$ and $\widetilde{u}(\widetilde{x}):=u(x)$ for every $\widetilde{x} \in \widetilde{\Omega}$. We observe that the $\widetilde{\mathscr{L}}$-propagation set of $\left(x_{0}, 0\right), \widetilde{P}_{\left(x_{0}, 0\right)}(\widetilde{\Omega})$, equals $\left.\mathscr{P}_{x_{0}}(\Omega) \times\right]-1,1\left[\right.$. Then $K \subseteq \operatorname{int} \mathscr{P}_{x_{0}}(\Omega)$ if and only if $\widetilde{K} \subseteq \operatorname{int} \widetilde{P}_{\left(x_{0}, 0\right)}(\widetilde{\Omega})$. By Theorem 1

$$
\sup _{\widetilde{K}} \widetilde{u} \leq C \widetilde{u}\left(x_{0}, 0\right)
$$

and the conclusion follows immediately.

\section{EXAMPLES}

In this Section we give two examples of operators for which we give Harnacktype inequalities that, to our knowledge, are new. In general, the main step in the application of our Theorem 1 is the characterization of the propagation set 
$\mathscr{P}\left(x_{0}, \Omega\right)$ of the operator $\mathscr{L}$. We recall that the Control Theory provides us with several tools useful for this problem. We refer, for example, to the book AS04, Chapter 8] by Agrachev and Sachkov.

\subsection{A Harnack inequality for the stationary Mumford operator.}

We consider the operator $\mathscr{L}=\partial_{x_{1}}^{2}+\sin \left(x_{1}\right) \partial_{x_{2}}+\cos \left(x_{1}\right) \partial_{x_{3}}$ in the set:

$$
\Omega=]-a, a\left[\times B(0, r) \subseteq \mathbb{R} \times \mathbb{R}^{2} .\right.
$$

$\left.x_{1} \in\right]-a, a\left[\right.$ where $a>\pi$, and $\left(x_{2}, x_{3}\right) \in B(0, r)$, the euclidean ball centered at 0 with radius $r>0$. This operator has been introduced by Mumford Mum94 in the study of computer vision problems. The relevant Harnack inequality of Theorem 1 takes the following form:

Theorem 3. Let $\Omega$ be the set introduced in (5.1), with $a>\pi$. For every compact set $K \subset \Omega$ there exists a positive constant $C=C(K, \Omega, \mathscr{L})$ such that

$$
\sup _{K} u \leq C u(0),
$$

for every non-negative solution $u$ of

$$
\partial_{x_{1}}^{2} u+\sin \left(x_{1}\right) \partial_{x_{2}} u+\cos \left(x_{1}\right) \partial_{x_{3}} u=0 \quad \text { in } \quad \Omega .
$$

Proof. In view of Theorem 1 we need only to prove that in this case the propagation set $\mathscr{P}(0, \Omega)$ agrees with $\Omega$. With this aim, we fix any point $z=\left(z_{1}, z_{2}, z_{3}\right)$ in $\Omega$, and we construct a $\mathscr{L}$-propagation path steering 0 to $z$. Note that, in our case, the vector fields defined in (1.2) are

$$
X=\partial_{x_{1}} \quad \text { and } \quad Y=\sin \left(x_{1}\right) \partial_{x_{2}}+\cos \left(x_{1}\right) \partial_{x_{3}} .
$$

We connect 0 and $z$ by a path $\gamma:[0, T] \rightarrow \Omega$ such that $\gamma^{\prime}(t)= \pm X(\gamma(t))$ in the first interval $\left[0, t_{1}\right]$, then $\gamma^{\prime}(t)=Y(\gamma(t))$ in the second interval $\left[t_{1}, t_{2}\right]$, and $\gamma^{\prime}(t)= \pm X(\gamma(t))$ in the third interval $\left[t_{2}, T\right]$, for $t_{1}, t_{2}, T$ such that $0 \leq t_{1} \leq t_{2} \leq T$ chosen as follows.

We set $\left.\left.\left.t^{*}=\arg \left(z_{2}, z_{3}\right) \in\right]-\pi, \pi\right] \subset\right]-a, a\left[\right.$, and we choose $t_{1}:=\left|t^{*}\right|$. If $t^{*}>0$, the function $\gamma(t)=(t, 0,0)$ is a solution of $\gamma^{\prime}(t)=X$, for $t \in\left[0, t_{1}\right], \gamma(0)=0$. If $t^{*}<0$ we consider $\gamma(t)=(-t, 0,0)$. In both cases, we have that $\gamma^{\prime}(t)= \pm X(\gamma(t))$. If $t^{*}=0$ we simply skip this step.

We next set $t_{2}=t_{1}+\sqrt{z_{2}^{2}+z_{3}^{2}}$, and we choose $\gamma$ such that $\gamma^{\prime}(t)=Y(\gamma(t))$ for $t_{1}<t<t_{2}$. Also in this case, if $\left(z_{2}, z_{3}\right)=(0,0)$, we skip this step. We conclude the construction of $\gamma$ by choosing $s^{*}=z_{1}-t^{*}, T=t_{2}+\left|s^{*}\right|$ and following the same method used in the first step. The path $\gamma$ then writes as follows. 


$$
\gamma(t)=\left\{\begin{array}{ccc}
( \pm t, 0,0) & \text { if } & 0 \leq t \leq t_{1}, \\
\left(t^{*},\left(t-t_{1}\right) \cos t^{*},\left(t-t_{1}\right) \sin t^{*}\right) & \text { if } & t_{1} \leq t \leq t_{2}, \\
\left(t^{*} \pm\left(t-t_{2}\right), z_{2}, z_{3}\right) & \text { if } & t_{2} \leq t \leq T .
\end{array}\right.
$$

Remark 4. The above construction can be reproduced to translated cylinders

$$
\left.\Omega_{y}=\right] y_{1}-a, y_{1}+a\left[\times B\left(\left(y_{2}, y_{3}\right), r\right) \subseteq \mathbb{R} \times \mathbb{R}^{2},\right.
$$

for every $y=\left(y_{1}, y_{2}, y_{3}\right) \in \mathbb{R}^{3}$. We find $\mathscr{P}\left(\left(y_{1}, y_{2}, y_{3}\right), \Omega_{y}\right)=\Omega_{y}$.

We point out that, on the other hand, the geometry of the propagation set $\mathscr{P}(0, \Omega)$ changes completely as the width of the interval $]-a, a[$ is smaller than $2 \pi$. For instance, if we consider the set

$$
\widetilde{\Omega}=]-\pi / 2, \pi / 2\left[\times B(0, r) \subseteq \mathbb{R} \times \mathbb{R}^{2},\right.
$$

we easily see that $\mathscr{P}(0, \widetilde{\Omega})=\widetilde{\Omega} \cap\left\{x_{3}>0\right\}$. This fact is in accordance with the invariance of the operator $\mathscr{L}$ with respect to the following left translation introduced in BL12. Denote $x=(t, z), y=(s, w) \in \mathbb{R} \times \mathbb{C}$. then

$$
x \circ y:=\left(t+s, z+w e^{i t}\right) .
$$

\subsection{A Harnack inequality for a degenerate Ornstein Uhlenbeck operator.}

We consider the operator $\mathscr{L}=\partial_{x_{1}}^{2}+x_{1} \partial_{x_{2}}$ in the set

$$
\Omega=]-a, a[\times]-b, b[
$$

for some positive $a$ and $b$.

As in the case of Mumford operator, Theorem 1 gives an elliptic Harnack inequality.

Theorem 5. Let $\Omega$ be the set introduced in (5.2). For every compact set $K \subset \Omega$ there exists a positive constant $C=C(K, \Omega, \mathscr{L})$ such that

$$
\sup _{K} u \leq C u(0),
$$

for every non-negative solution u of

$$
\partial_{x_{1}}^{2} u+x_{1} \partial_{x_{2}} u=0 \quad \text { in } \quad \Omega .
$$


Proof. We prove that, also in this case, the propagation set $\mathscr{P}(0, \Omega)$ agrees with $\Omega$. The vector fields defined in (1.2) are

$$
X=\partial_{x_{1}} \quad \text { and } \quad Y=x_{1} \partial_{x_{2}} .
$$

We choose an integral curve $\gamma$ such that $\gamma^{\prime}(t)= \pm X(\gamma(t))$ in some intervals. A curve like that writes as $\gamma(t)=\left(\widetilde{x}_{1} \pm t, \widetilde{x}_{2}\right)$. In particular, we will use the field $X$ to increase or decrease the first coordinate $x_{1}$. In some other intervals we choose $\gamma^{\prime}(t)=Y(\gamma(t))$. Such a curve writes as $\gamma(t)=\left(\widetilde{x}_{1}, \widetilde{x}_{2}+\widetilde{x}_{1} t\right)$. In this case, we rely on the sign of $\widetilde{x}_{1}$ to increase or decrease the second component $x_{2}$. We prefer not to give the details of the construction and to refer to the following figure.

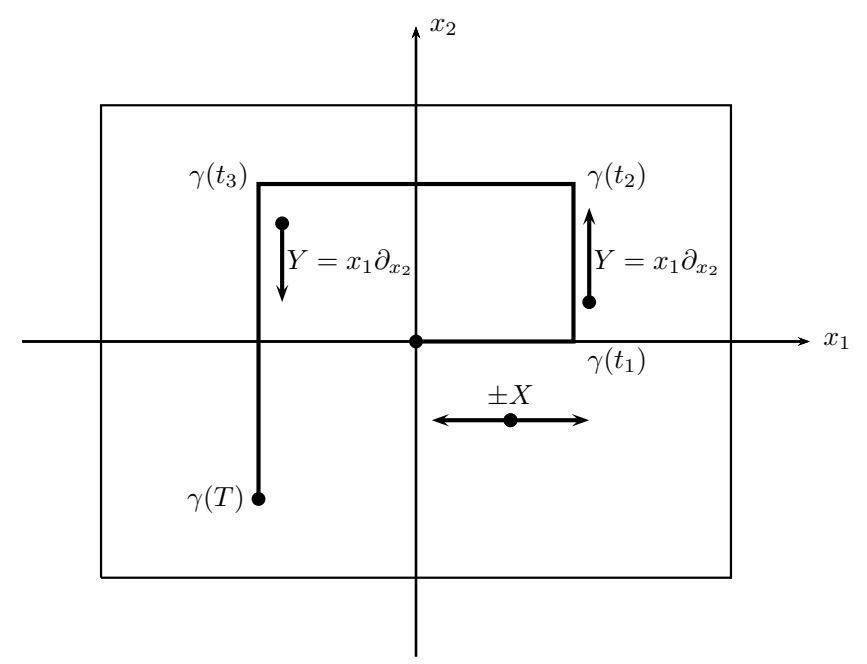

Remark 6 . The above result fails as

$$
\Omega=] a_{1}, a_{2}[\times]-b, b\left[\subseteq \mathbb{R}^{2},\right.
$$

and $a_{1}$ and $a_{2}$ have the same sign. In particular, if $a_{1}$ and $a_{2}$ are both positive, and we consider $x_{0}=\left(\frac{a_{1}+a_{2}}{2}, 0\right)$, we have $\mathscr{P}\left(x_{0}, \Omega\right)=\Omega \cap\left\{x_{2}>0\right\}$. On the contrary, if $a_{1}$ and $a_{2}$ are both negative, we have $\mathscr{P}\left(x_{0}, \Omega\right)=\Omega \cap\left\{x_{2}<0\right\}$.

\section{ACKNOWLEDGMENTS}

The authors have been partially supported by the Gruppo Nazionale per l'Analisi Matematica, la Probabilità e le loro Applicazioni (GNAMPA) of the Istituto Nazionale di Alta Matematica (INdAM). 


\section{REFERENCES}

[Ama79] K. Amano. Maximum principles for degenerate elliptic-parabolic operators. Indiana Univ. Math. J., 28(4):545-557, 1979.

[AS04] A. A. Agrachev and Y. L. Sachkov. Control theory from the geometric viewpoint, volume 87 of Encyclopaedia of Mathematical Sciences. Springer-Verlag, Berlin, 2004. Control Theory and Optimization, II.

[Bau66] H. Bauer. Harmonische Räume und ihre Potentialtheorie. Ausarbeitung einer im Sommersemester 1965 an der Universität Hamburg gehaltenen Vorlesung. Lecture Notes in Mathematics, No. 22. Springer-Verlag, Berlin-New York, 1966.

[BL12] A. Bonfiglioli and E. Lanconelli. Matrix-exponential groups and Kolmogorov-FokkerPlanck equations. J. Evol. Equ., 12(1):59-82, 2012.

[BLU02] A. Bonfiglioli, E. Lanconelli, and F. Uguzzoni. Uniform Gaussian estimates for the fundamental solutions for heat operators on Carnot groups. Adv. Differential Equations, 7(10):1153-1192, 2002.

[BLU07] A. Bonfiglioli, E. Lanconelli, and F. Uguzzoni. Stratified Lie groups and potential theory for their sub-Laplacians. Springer Monographs in Mathematics. Springer, Berlin, 2007.

[Bon69] J.-M. Bony. Principe du maximum, inégalite de Harnack et unicité du problème de Cauchy pour les opérateurs elliptiques dégénérés. Ann. Inst. Fourier (Grenoble), 19(fasc. 1):277-304 xii, 1969.

[CC72] C. Constantinescu and A. Cornea. Potential theory on harmonic spaces. Springer-Verlag, New York-Heidelberg, 1972. With a preface by H. Bauer, Die Grundlehren der mathematischen Wissenschaften, Band 158.

[CMP15] C. Cinti, S. Menozzi, and S. Polidoro. Two-sided bounds for degenerate processes with densities supported in subsets of $\mathbb{R}^{N}$. Potential Anal., 42(1):39-98, 2015.

[CNP10] C. Cinti, K. Nyström, and S. Polidoro. A note on Harnack inequalities and propagation sets for a class of hypoelliptic operators. Potential Anal., 33(4):341-354, 2010.

[KL04] A.E. Kogoj and E. Lanconelli. An invariant Harnack inequality for a class of hypoelliptic ultraparabolic equations. Mediterr. J. Math., 1(1):51-80, 2004.

[Mum94] D. Mumford. Elastica and computer vision. In Algebraic geometry and its applications (West Lafayette, IN, 1990), pages 491-506. Springer, New York, 1994.

[Rud87] W. Rudin. Real and complex analysis. McGraw-Hill Book Co., New York, third edition, 1987.

Dipartimento di Ingegneria dell'Informazione, Ingegneria Elettrica e Matematica Applicata, Universitì degli Studi di Salerno, Via Giovanni Paolo II, 132, IT-84084 FisCIANO (SA) - ITALY

E-mail address: alessia.kogoj@gmail.com

Dipartimento di Scienze Fisiche, Informatiche e Matematiche - Università di Modena

e Reggio Emilia - via Campi 213/B, IT-41125 Modena - Italy

E-mail address: sergio.polidoro@unimore.it 